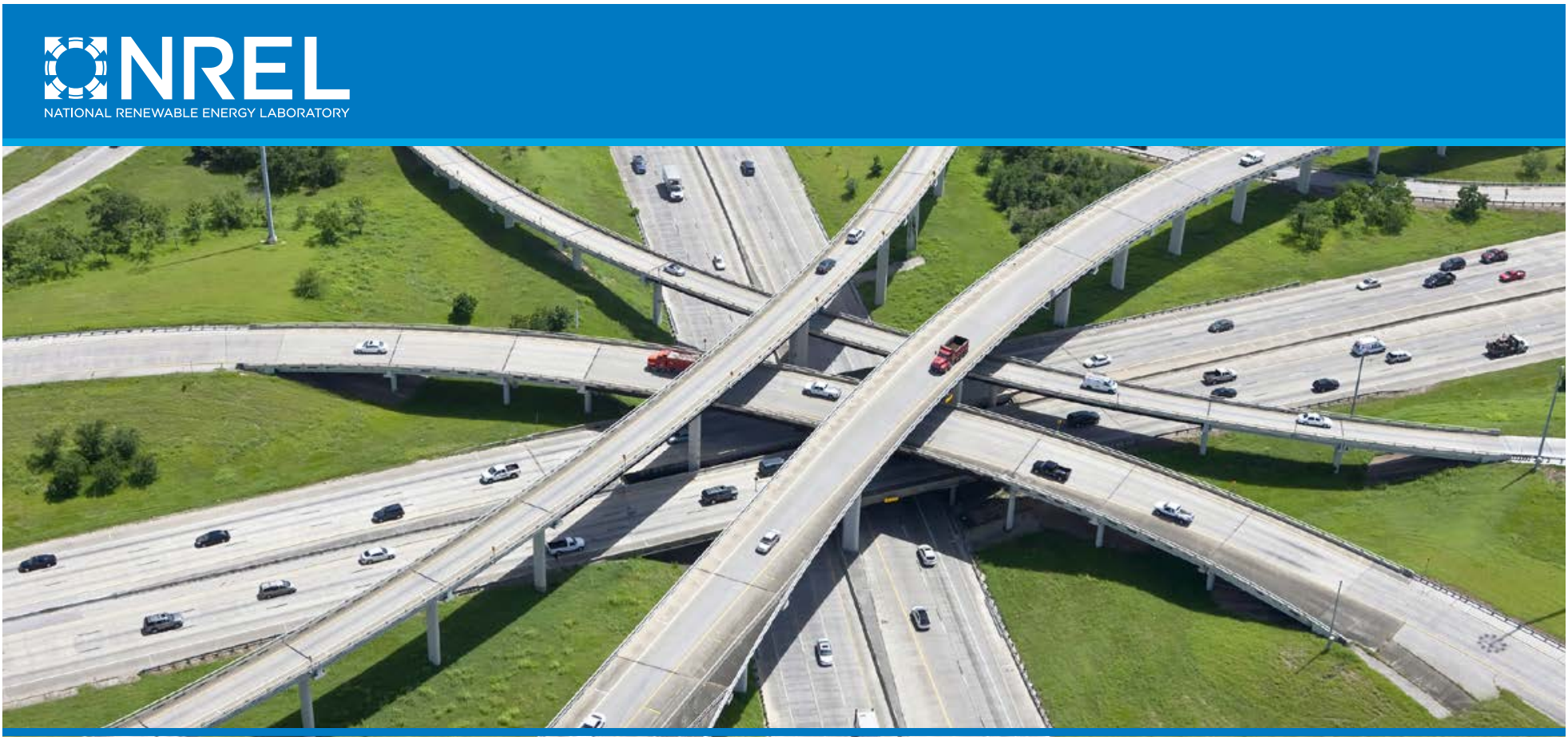

Photo from iStock 13592574

\title{
DRIVE Analysis Tool Generates Custom Vehicle Drive Cycles Based on Real-World Data
}

Understanding vehicle usage plays a fundamental role in assessing the performance of new vehicle technologies, leading to more informed decision making, better test procedures, more successful designs, and lower manufacturing and operating expenses. The Drive-Cycle Rapid Investigation, Visualization, and Evaluation (DRIVE) analysis tool produces representative, testable drive cycles at record speed from large amounts of vehicle data gathered via onboard logging devices. Developed by the National Renewable Energy Laboratory (NREL), DRIVE uses GPS and controller area network data to characterize vehicle operation and produce custom vehicle drive cycles based on real-world activity, analyzing thousands of hours of data in a matter of minutes.

\section{From Raw Data to Custom Drive Cycles}

DRIVE quickly filters large sets of raw data, removing erroneous data points and repairing missing data sections before performing analyses covering 168 unique vehicle drive cycle metrics. Upon filtration and analysis, the tool generates custom representative drive cycles from "ideal" sections of filtered data using specialized statistical clustering methods. The duration of these representative drive cycles, which aim to match the fuel economy and performance characteristics of the source data, can be specified via user-defined time limits, allowing for a wider range of testing and modeling applications.

\section{Analysis and Comparisons}

In addition to DRIVE's ability to generate custom-duration representative drive cycles from raw data, the tool is capable of comparing vehicle operation to industry standard test cycles and can even select a representative standard cycle from its built-in library. Comparing the filtered data across the calculated drive cycle metrics allows the tool to select the standard cycle with the best fit.

Algorithms mimic the logic and expertise of a human engineer, cutting testing and analysis time by days or weeks while establishing a repeatable process and making information accessible through a simple user interface. Additional output results range from simple tabulated summary statistics to Google Earth route maps, providing information that has enough depth for scientific applications but is accessible to users without technical backgrounds. 


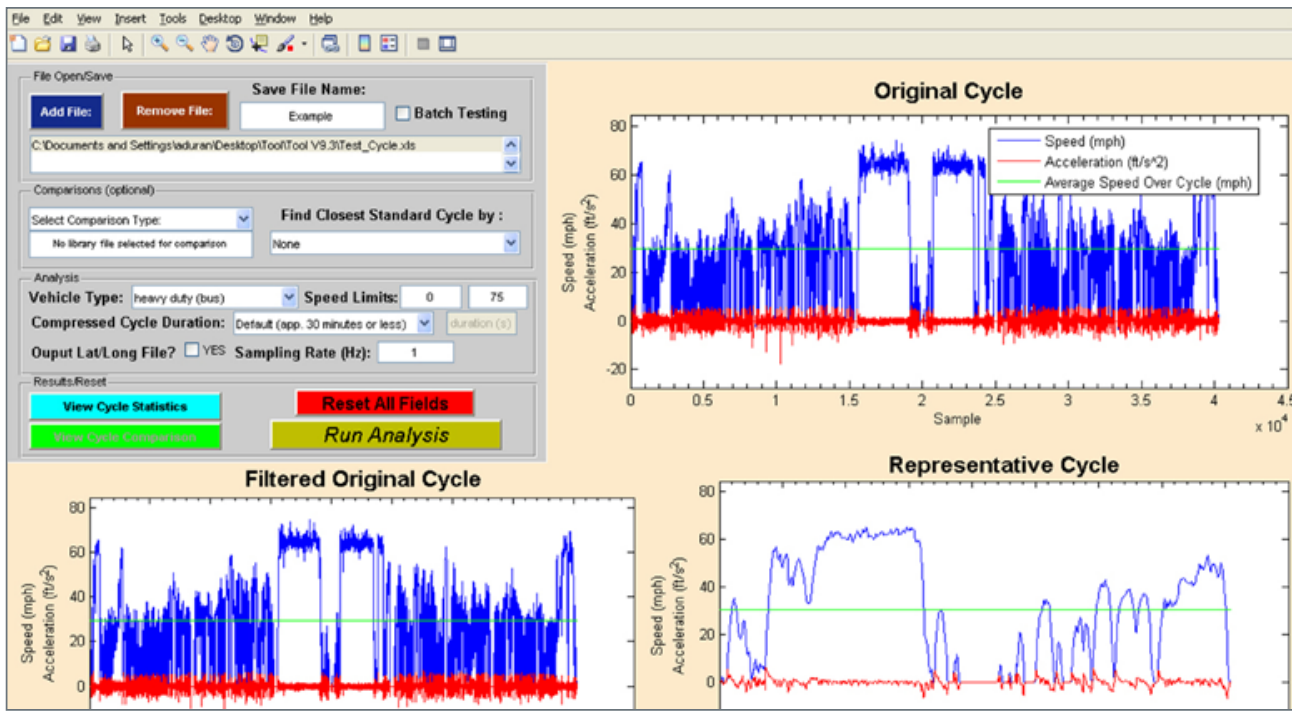

This screenshot of DRIVE's user interface displays speed versus time plots of source data, cleaned data, and custom drive cycle data.

\section{Users and Applications}

Designed for use with a full range of vehicle types and sizes, DRIVE can benefit a variety of users. For example:

- Fleet managers can use the tool to make educated investment decisions by determining, in advance, the payback period for a given technology.

- Vehicle developers can use DRIVE in conjunction with vehicle modeling tools for more efficient and accurate vehicle component sizing, reducing technology cost and development time.

- DRIVE can help manufacturers ensure designs are based on real-world vehicle operation, supplying information necessary for performing vital development tasks, such as sizing electric motors in a hybrid vehicle configuration or optimizing battery storage in an electric vehicle.

- For researchers, DRIVE provides statistical results and test cycles that can lead to improved models and more precise experiment designs.

- For regulators, a better understanding of the way people drive can help guide policy regarding vehicle emissions, incentives, and fuel economy test procedures.
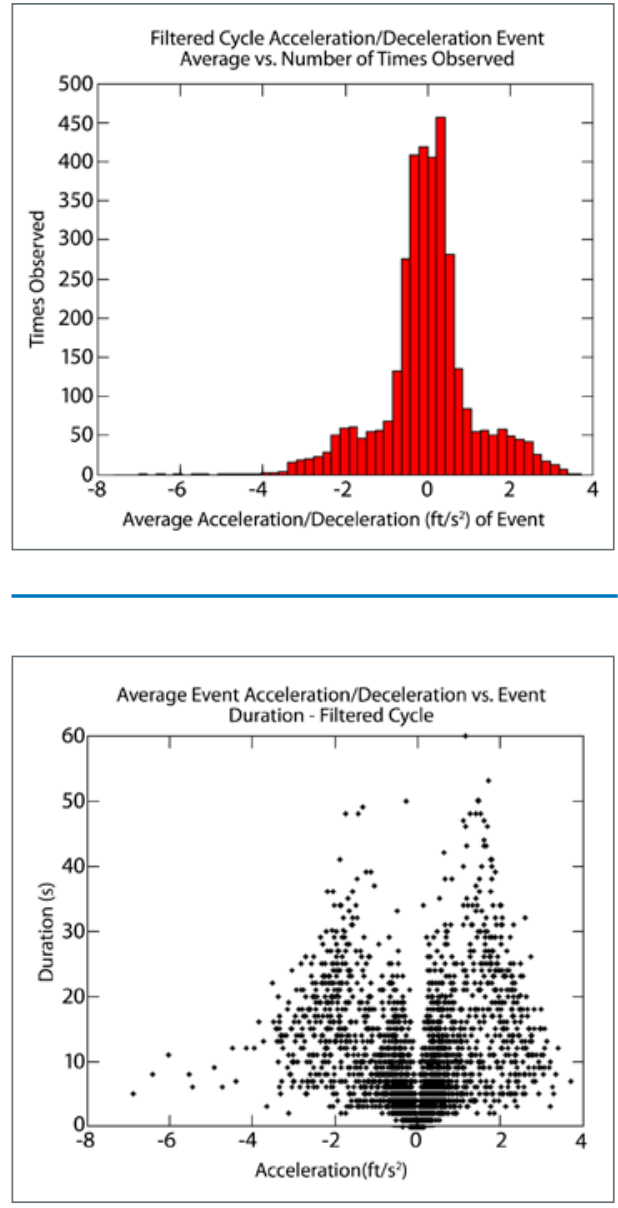

In addition to the existing data visualization features shown here, DRIVE outputs can be used in conjunction with commonly available software packages to generate more detailed data visualizations.

\section{Obtaining a Copy of DRIVE}

DRIVE is available for a 30-day trial at no cost. NREL also offers two licensing options: Pay an upfront fee for a copy of the software, or receive the software for free via a collaborative data-sharing agreement. Contact Adam Duran at 303-275-4586, adam.duran@nrel.gov, for more information.

\section{About NREL's Transportation R\&D}

Dedicated to renewable energy and energy efficiency, NREL is at the forefront of R\&D for tomorrow's sustainable transportation solutions. NREL's innovative approach helps government, industry, and other research partners develop high-performance, low-emissions, fuel-efficient vehicles, components, and systems.
National Renewable Energy Laboratory 15013 Denver West Parkway Golden, CO 80401

303-275-3000 • www.nrel.gov
NREL is a national laboratory of the U.S. Department of Energy, Office of Energy Efficiency and Renewable Energy, operated by the Alliance for Sustainable Energy, LLC. NREL/FS-5400-54507 • April 2013

Printed with a renewable-source ink on paper containing at least $50 \%$ wastepaper, including $10 \%$ post consumer waste. 